\title{
Grass Genomic Synteny Illuminates Plant Genome Function and Evolution
}

\author{
Jeffrey L. Bennetzen • Mingsheng Chen
}

Received: 1 October 2008 / Accepted: 21 October 2008 / Published online: 12 November 2008

(C) Springer Science + Business Media, LLC 2008

\begin{abstract}
The genic colinearity of grass genetic maps, physical maps, and contiguous genomic sequences has been a major contributor to almost two decades of study into the structure and evolution of grass genomes. This research has led to the discovery of all of the major phenomena responsible for the rapid evolution of flowering plant genomes. These processes include polyploidy, transposable element (TE) amplification, TE-driven genome rearrangement, and DNA removal by unequal homologous recombination and illegitimate recombination. The great variety in angiosperm genome structure is largely an outcome of differences in the specificities, frequencies, and amplitudes of these common genome-altering processes. Future emphasis now needs to shift to harnessing an even broader range of studied species, and to use this phylogenomic perspective to uncover the nature and functions of the genes that are shared by particular lineages and those that set each individual species apart as a unique biological entity.
\end{abstract}

Keywords Comparative genomics - Genome evolution · Microcolinearity $\cdot$ Recombination · Transposable elements

\footnotetext{
J. L. Bennetzen $(\bowtie)$

Department of Genetics, University of Georgia,

Athens, GA 30602-7223, USA

e-mail: maize@uga.edu

M. Chen

State Key Laboratory of Plant Genomics, Institute of Genetics and Developmental Biology, Chinese Academy of Sciences, Beijing 100101, China
}

\section{Introduction}

Two of the central questions in biology are what are the genetic foundations that underlie the similarities between different species or individuals within a species and what are the genetic variations responsible for the observed differences. Even from the first days of comparative genomics, it was surprising to many that humans, fruit flies, nematodes, and yeast shared a large percentage of their genes $[2,85]$. Given our human-centered worldview, it was to be expected that many would be shocked by the fact that humans and our closest surviving relative, the chimpanzee, share $\sim 98 \%$ sequence identity and an even higher similarity in gene content [81]. In plants, haplotypic differences in genome sequence within a species like maize can greatly outstrip these interspecies primate variations. Of course, not all sequence change is equally significant, and work on the evolution of maize has shown that tiny changes in regulatory loci can dramatically alter morphology and behavior [24].

The field of comparative genomics was founded on the idea that comprehensive analyses and comparison of whole genomes could uncover the essential conserved, and the importantly variable, components of any set of genomes. In plants, this comparative analysis proved to be particularly challenging for several reasons, including (1) the small number of species that were investigated, (2) their large and complex genomes, and (3) their high rate of structural rearrangement. The observation that closely related plants sometimes exhibited regions of DNA-marker colinearity [11, 38] provided a key point of constancy in these comparisons because genetic map relatedness was simple to determine with robust techniques that were not dramatically affected by genome size or the overall quality of the genetic toolkit for that species [8]. The first demonstrations of microcolinearity (also called microsynteny) by comparative 
sequencing of orthologous chromosomal regions [17, 84] indicated that overall genomic similarity could be converted into very local analyses of the evolved structure and function of genes that were all derived from a known ancestral locus at attributable dates. Hence, both whole genome and individual gene analyses could be made in a comprehensive manner across many species, as first proposed and illustrated in the grasses.

Another enduring question that was illuminated by a comparative genome analysis strategy was the nature of the DNA in the eukaryotic nucleus. For many decades, it has been known that nuclear genomes vary dramatically in size, even between closely related species, and the mystery behind this "unexplained" or presumed "excess" DNA was termed the "C-value paradox" by Thomas [82]. Research in flowering plants, where the differences in nuclear DNA content varies more than one thousand fold, has explained this $\mathrm{C}$-value variation [10]. We now know that the differences in $\mathrm{C}$-value across flowering plants are very dynamic outcomes of occasional polyploidy along with great variability in transposable element (TE) amplification and processes for DNA removal. However, we do not know how often these changes (especially those caused by TEs or other small indels) generate selectable variation that can lead to changed capabilities within a species or to speciation.

This plant genome review will discuss the discovery of genomic colinearity and synteny, its biological origins, its numerous exceptions, and its uses for genome analysis. We will focus on the grasses because this is our area of greatest expertise and because this is also the source of the most comprehensive sets of data and analyses in plants. We have every reason to believe that much of what is discussed herein for the grasses will also be true in other plants, and in more distantly related eukaryotes.

\section{Genetic map colinearity in the grasses: rules and exceptions}

\section{The crop circle}

The first comparative genetic map in the grasses was a miniscule maize::sorghum comparison in a study meant to test whether restriction fragment length polymorphism (RFLP) markers generated in one species (e.g., maize) could be used to help generate a genetic map in other species (e.g., sorghum) [38]. This project indicated that maize RFLP probes could be used routinely for species as far distant as foxtail millet, a lineage that last shared a common ancestor with the maize lineage about 30 million years ago (mya) [48]. Serious grass genome comparisons were then generated by expert mapping labs, especially the
Gale and Tanksley groups [3, 4, 20]. These studies indicated a good deal of similarity in gene content and colinearity, with a low frequency of small and large exceptions. Moore et al. [67] provided a major conceptual leap when they identified a series of conserved grass genome segments and then assembled them into a comparative circle map.

The comparative circular map of the grasses, also known as the crop circle, has allowed identification of the major rearrangements that differentiate grass genomes, and has provided insight into the timing of these events during grass descent from a common ancestor more than 50 mya. As shown in Fig. 1, gene order and telomere location are largely conserved at this scale, although the number of chromosomes is quite variable across species. Maize yields two concentric circles, suggestive of a whole genome polyploidy event, which has been confirmed by extensive orthologous DNA sequence analysis [80]. Two specific translocations, shown at 3 o'clock and 7 o'clock on the circle map, are shared by all investigated members of the Panicoid subfamily but not by rice or the Triticeae. Most of the other detected rearrangements are inversions that are limited to only one or two of the species depicted (Fig. 1).

In addition, this circular map allowed the easily visualized (and thus conceptualized and transmitted) discovery that some important genes involved in domestication or other important traits appeared to be the same orthologous loci across multiple grass species (Fig. 1) [72]. This, in turn, helped encourage the use of surrogate plant chromosomes like the relatively small genome of rice to assist in the map-based cloning of genes in large-genome species like barley, wheat, or sugarcane [14, 33, 19].

Three major conclusions that were clear from the comparative circular maps were (1) the relatively low frequency of large genomic rearrangements, (2) the presence of inversions, translocations and duplications, and (3) the uneven distribution of such events, with many at boundaries near current centromere locations (Fig. 1). Among the many issues not resolved by this analysis, however, was whether the frequencies of these major rearrangements were in any way predictive or mechanistically similar to the frequencies and types of local rearrangements. More detailed physical and genetic maps would be needed to address these questions.

Physical maps, genetic maps and their comparison

Early studies by comparative genetic mapping revealed the extent of conservation of gene content and gross gene order among different grass species, but did not give many insights into the likelihood or nature of small rearrangements. In these first studies [38], it was observed that most maize RFLP probes hybridized strongly to sorghum DNA, 
Fig. 1 Synteny of five crop genomes. Different color bars represent the chromosomes in different grass genomes, with their telomeres indicated by red triangles. Arrows show rearrangements relative to rice. Arrows with a single arrowhead are translocations, and those with two arrowheads are inversions. Arrows at $3 o^{\prime}$ clock and 7 $o^{\prime}$ clock indicate rearrangements that are shared by the subfamily Panicoideae (foxtail millet, sorghum, and maize). Dotted bars indicate regions where insufficient data were available at the time of the analysis undertaken by Gale and Devos [30]. The dotted internal line indicates a duplication shared by chromosomes 11 and 12 of rice [69]. Red dots are orthologous genes controlling semi-dwarf phenotypes that are located on rice chromosome 3 , wheat chromosome 4 and maize chromosome 1 [22, 73]. pt Part of a chromosome.

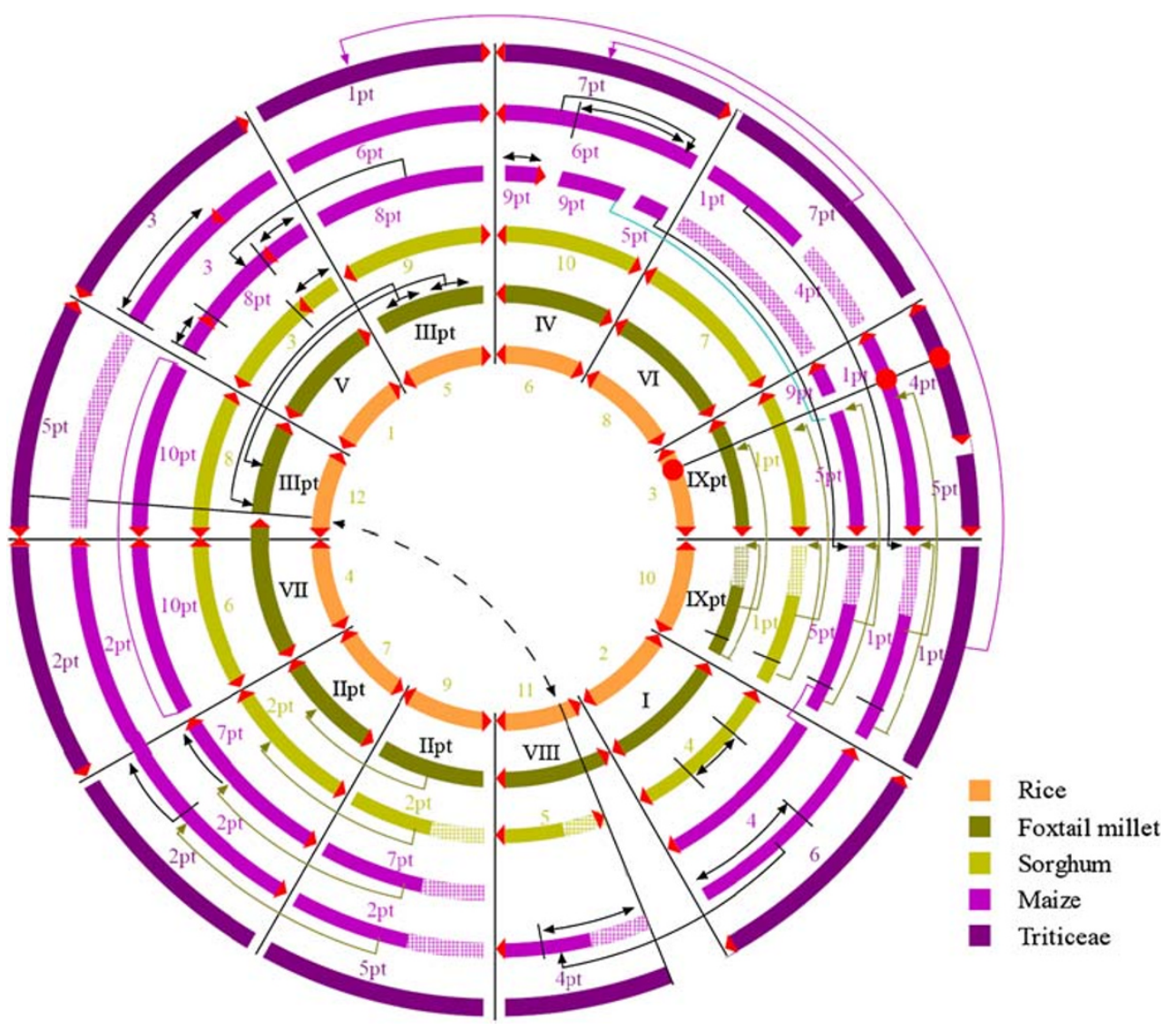

but the repetitive DNA sequences in maize usually did not hybridize to sorghum. This suggested that repetitive DNA sequences evolved much faster than genes, and that heterologous probes could thus provide some advantages over homologous probes from a repeat-rich genome.

Comparative genetic mapping between closely related grasses, such as sorghum and sugarcane, whose separate lineages diverged from each other about 8 mya, show striking map colinearity [35]. In contrast, detailed comparative genetic mapping among more distantly related species, such as maize and rice, identified numerous chromosomal rearrangements, such as telomeric fusions, nested insertions, inversions and translocations [92], although about $2 / 3$ of these genomes appeared to still be colinear. Many of the detected rearrangements were confirmed by comparative physical mapping, such as (from a rice perspective) the fusion of rice chromosomes 3 and 10 and chromosomes 7 and 9 into single chromosomes in the Panicoideae lineage [88]. In addition, comparative physical mapping also uncovered the ancient grass genome duplication shared by maize [88], wheat [77], and other grasses [71].

Comparative physical mapping between sorghum and rice revealed different genome components with very different degrees of microcolinearity. In euchromatic regions, where most meiotic recombination occurs, greater microcolinearity was observed; however, less microcolinearity was observed in recombination-poor heterochromatin, such as pericentromeric regions [12]. This phenomenon was also apparent in comparison of homoeologous chromosomal regions in rice derived from the ancient duplication at the origin of the grasses, where little colinearity was retained in pericentromeric regions [83]. In addition, the heterochromatic regions of sorghum have been preferentially expanded relative to rice, as compared to euchromatic regions [51]. Future detailed studies of microcolinearity in heterochromatin are needed to uncover the dynamics and mechanisms for macroand micro-rearrangements in these crossover-deficient parts of grass genomes $[61,63]$.

\section{Microcolinearity}

Across the grasses (and a bit beyond)

Even from the start, comparisons of genomic sequence in orthologous regions of different grass species examined a very large time frame, such as rice versus sorghum [17] or rice versus various Triticeae [28, 37, 26], all comparisons where the investigated species last shared a common ancestor $\sim 50$ mya. In this time frame, the sequences between genes appeared to be completely different, although very tiny "conserved non-coding sequences" 
(CNS) were later discovered [45, 36]. Even introns of orthologous genes, although largely consistent in location across all flowering plants, contained obvious conserved sequences only at the boundaries needed to specify appropriate RNA processing. Hence, the general conclusion could be reached that anything still conserved after 50 million years of grass genome divergence was likely to have an important function.

Gene content and order, on the other hand, were mostly conserved on segments of a few dozen to a few hundred $\mathrm{kb}$ even after 50 million years of independent grass genome evolution. Comparisons to rice have been particularly useful in this regard because (1) it is evolutionarily quite distant from the other important grasses like maize, wheat, barley, and sorghum [48], (2) it has a relatively small genome ( $400 \mathrm{Mb})$ with a high gene density, (3) it's genome was an early target for comprehensive sequence analysis [41], and (4) it has proven to be more stable visà-vis small local rearrangements than other grasses like maize, sorghum, wheat or barley [9].

In the most comprehensive comparisons to date, between rice and two panicoid grasses, sorghum and maize, the frequency of gene movement over the last fifty million years was calculated as at least 5\%, and possibly as high as $25 \%$, between sorghum and rice [53]. This number does not include the gain or loss of tandemly repeated gene copies, a very common phenomenon in all grass lineages investigated. Most of the genic rearrangements in maize compared to either rice or sorghum are apparent gene losses on one of two maize homoeologues [40,53], an expected outcome of the polyploidization event about five mya that gave rise to the Zea lineage [80]. However, too little data yet exist to identify possible subtle patterns in types of rearrangement. Moreover, rearrangements involving genes are likely to be under selective pressure, so the events currently observed in any species are a combined outcome of those events that have occurred, minus those that were subsequently removed by chance or by selection against some specific changes.

In more distant comparisons, with longer ancestral divergence times, colinearity across orthologous regions appears to be much more rare than within the grasses. In the rice flatsedge, Cyperus iria, the near-adjacent $\mathrm{Sh} 2$ and $\mathrm{Al}$ homologues appear to be conserved in order and orientation, but one of the two genes in between in the grasses is missing in the sedge (A. Pontaroli and J. Bennetzen, unpub. obs.). However, this is the only comparison that has been done to the grasses in this $~ 110$-million-years-of-divergence window [13]. Similarly, Musa (e.g., banana) genomes show some colinearity with the grasses after $>115$ million years of divergence from their last shared ancestor, but more than $50 \%$ of the annotated genes were non-colinear in a comparison to rice [56]. With even more distant comparison to the eudicots, $>220$ million years of independent descent, only rare segments of genic colinearity are observed at either full genome or local genome scales [58].

The most frequent type of structural change in all investigated angiosperm nuclear genomes has been observed to be the differential insertion and subsequent instability of transposable elements (TEs). In large-genome species like maize and barley, most of the DNA between genes is comprised of TEs, especially long terminal repeat (LTR) retrotransposons [78, 86, 89, 75]. These elements transpose by reverse transcription of an RNA transcript and insertion of the resultant DNA, so transposition does not involve excision. Because LTR retrotransposons make up more than $50 \%$ of most or all large flowering plant genomes and their high content varies somewhat proportionally with angiosperm genome size, it is clear that these TEs are the most important factor responsible for genome size variation in flowering plants [10]. Because these TEs (and all other unselected DNAs) are fragmented and removed so rapidly by accumulated small deletions (see below), all of the insertions appear to be very recent, usually within the last 2-6 million years [87]. This accounts for the near-complete lack of homology of the intergenic regions in orthologous genome segments with grass lineages that last shared a common ancestor more than 50 mya.

We currently lack a vocabulary to precisely describe the degree of conservation of genic content and colinearity between any two species, much less across multiple species, although a gene-pair conservation terminology is currently in development (L. Feng and J. Bennetzen, unpub. res.). However, it is clear that some lineages are very unstable (e.g., pearl millet, sorghum, maize) and others are much more stable (e.g., rice and foxtail millet) at the level of compared genetic maps and/or microcolinearity $[23,75,9,40]$. We do not yet know the reasons for these differences, nor whether high conservation at one scale (e.g., genetic map) in any way correlates with high conservation at other scales (e.g., physical map or microcolinearity). It is clear, though, that certain types of gene rearrangement are rare (e.g., movement of a gene to a wholly different chromosome) while others are relatively common (tandem duplication, deletion or inversion of small genic segments).

Analysis of microcolinearity and gene content conservation at long time frames has the advantage of the accumulation of multiple events for analysis, but this is more than counterbalanced by three negative aspects of concentrating on such ancient rearrangements. First, natural selection has had a great deal of time to remove any events that had even a minor organismal disadvantage, so one only observes certain classes of tolerated or advantageous events that might not be proportional to the true spectrum of $d e$ 
novo rearrangements. Second, the components of the genome responsible for the rearrangement have had ample time to decay into a state where they are invisible to current annotation approaches. And, third, individual events may be buried underneath second, third or more layers of events at the same location. For all of these reasons, investigations of orthologous regions in closely related lineages are justified, and are expected to be "there to discover" because of the relatively high rate of local chromosomal rearrangement in the grasses.

Colinearity dynamics within a $0-15$ million year window of grass genome evolution

Orthologous sequence comparisons across short time frames has the potential to reveal both the rate and the mechanisms for disruption of colinearity. In a sequence comparison of the adh1-orthologous regions of maize and sorghum, two species that last shared a common ancestor about 12 mya [80], a 212-kb maize sequence was found to be largely collinear with a $66-\mathrm{kb}$ sorghum sequence [84]. The more than three-fold size difference is mainly due to nested LTR retrotransposon insertions in the maize genome $[78,84]$. In the original annotation, orthologs of nine maize genes were detected in the sorghum region in perfect colinear order; however, three additional genes in this sorghum segment were not found in the maize adhl region. In subsequent analyses, one of the "missing" maize genes was found to be located in the adhl-homoeologous region of maize [40]. This has now turned out to be a routine situation in the maize genome, where two maize segments represent each sorghum region due to a polyploidy event in the Zea lineage within the last few million years [80]. Gene deletion (usually of only one homoeologous copy) subsequent to polyploidization has now reduced the originally doubled copy number of genes $(2 \times)$ to less than $1.5 \times$ [53]. The other two non-colinear genes in the adh1-orthologous regions of sorghum are found elsewhere in the genomes of maize and other grasses and are hypothesized to have been caused by the insertion of two unlinked genes, either as two subsequent events or by a single event involving three chromatids. In dramatic contrast, a comparison of the adh1orthologous regions between sorghum and sugarcane, both gene colinearity and strong homology of non-coding regions were observed [42], indicating greater stability in these lineages over this shorter ( $\sim 8$ million year) time frame of divergence.

In at least some genomes, polyploidization is followed by extensive genomic change resulting in the silencing and elimination of duplicated genes [1]. In grasses, polyploidy has been a recurrent theme, with many lineages exhibiting full genome duplications over the last few million years. Local sequence comparisons in these species, such as maize
$[40,53,64]$, wheat $[27,46,90,34,16,15]$ and sugarcane [42], have revealed interesting features of gene and genome evolution in recent polyploids. LTR retrotransposon amplification and altered regulation (e.g., silencing) or loss of duplicated genes are repeated themes. Inactivation and eventual elimination of duplicated genes can be mediated by altered epigenetic regulation, deletions, TE insertions, and/or point mutations causing premature stop codons.

Some evidence suggests that specific alterations recur in independent polyploidizations in wheat [27, 46] and Brassica napus [59]. However, most eventually fixed changes do not occur instantly in post-polyploid genome rearrangements, at least not in maize. In adhl-homoeologous regions, for instance, fragments of partially deleted genes remain, indicating the incomplete status of removal several million years after polyploidy, and showing that these gene losses are primarily by the accumulation of multiple small deletions [40]. Another example of reasonably stable polyploid gene copies comes from a comparative study of the adh1orthologous regions of maize, sorghum and sugarcane [42]. The two sugarcane homoeologous haplotypes show perfect genic colinearity. In addition, two maize homoeologous regions yielded the same gene content, order and orientation as in sugarcane. Our data on comparative analysis in the Oryza genus also reveals excellent stability of polyploid genomes formed less than two million years ago (Chen et al., unpub. res.).

The Oryza genus contains about 24 species that belongs to ten different genome types [31]. A project, entitled the Oryza MAP Alignment Project (OMAP), was launched to build a framework for comparative biology in the Oryza genus [93]. Representative species, ranging from closely related species/subspecies, such as those with AA genomes, which diverged from their common ancestor less than a million years ago, to more distantly related species, such as $O$. brachyantha and $O$. granulata, whose ancestors diverged about 10 mya, were chosen for bacterial artificial chromosome (BAC) library construction, BAC end sequencing, and physical map construction [5, 49]. The initial analyses revealed excellent gene colinearity both in their physical maps [50] and in sequence comparisons [96]. Genome size variations in the Oryza genus were found to be mainly caused by lineage-specific amplifications of LTR retrotransposons $[74,6]$. Our systematic comparative analysis of the sequence of the MONOCLUM1-ortholgous regions across the Oryza genus not only revealed high gene colinearity but also identified new genes that appear to have originated de novo in the AA genomes (Fig. 2 and Chen et al., unpub. res.), which highlights the advantage of multiple species comparisons.

Intraspecific local sequence comparisons have also identified interesting features of grass genome structure 
Fig. 2 Microcolinearity in the MONOCULM1-orthologous regions across the Oryza genus. Black boxes represent genes. Red boxes indicate retrotransposons. Fuchsia boxes symbolize DNA transposons. Orthologous genes are connected by lines.

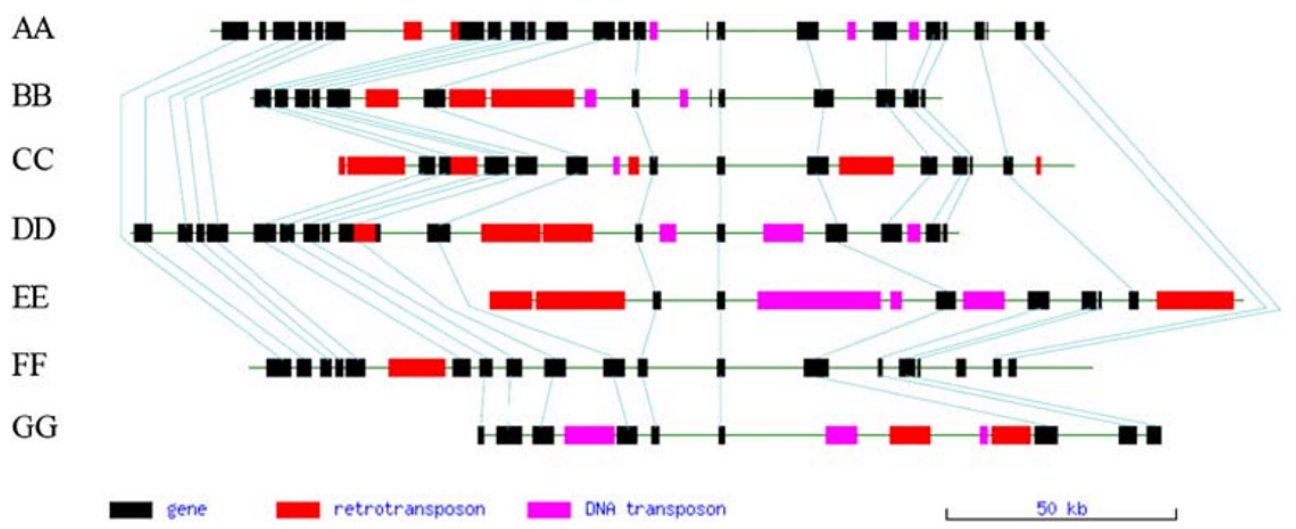

and evolution. A detailed sequence comparison of the bronze region of maize inbred lines $\mathrm{McC}$ and $\mathrm{B} 73$ found that LTR retrotransposon clusters differed one hundred percent in location relative to the genes in the bronze region between these two lines [29]. This suggests an amazingly rapid process both for TE insertion and for removal of ancestral TEs. In addition, the first annotation of these two regions suggested that the genes themselves differed between these lines in this region. An apparent four-gene cluster was detected in $\mathrm{McC}$ but not in the orthologous position in B73 [29]. Later, these sequences were found to be comprised of four gene fragments within Helitrons, a new type of eukaryotic transposon [44, 54, 52, 68]. This phenomenon resembles Pack-MULEs, a type of TE first named and comprehensively described in rice, that also capture and mobilize gene fragments [43]. Although neither Helitrons nor Pack-MULEs usually mobilize intact genes, they do commonly acquire more than one gene fragment in the same element. When transcribed, these internal fragments are often fused (via intron processing) into transcripts that could encode novel protein products [43, 68]. This process of exon shuffling, first proposed by Gilbert [32] as the reason for the existence of introns, could be creating new genes in plants at an amazing rate. The maize nuclear genome, for instance, has more than 4,000 Helitrons that contain inserted gene fragments [68] (L. Yang and J. Bennetzen, unpub. res.). However, there is not yet a proven case of any of these Helitron- or Pack-MULE-generated "new" genes having actually acquired a genetic function essential to its host. Given the rapid rate of unselected DNA loss from plant genomes (see below), it is unlikely that conversion of these chimeric gene candidates into true genes will occur commonly, but even rates as low as one in a million would be significant. Other than the standard route of gene duplication, which primarily creates subfunctionalized or (rarely) mildly modified new gene functions (reviewed in [39]), there is no known aggressive process for the generation of new genes. Perhaps PackMULEs and Helitrons will eventually be proven to provide this process. At the very least, we expect to see the discovery of more cases of TE components being co-opted for organismal functions in plants, as in the recent identification of transcription factors in Arabidopsis derived from the Mutator transposase [57].

Even if TE-vectored gene fragments are rarely if ever true genes with a selected host function, they certainly are a complication to genome annotation. Even without internal gene fragments, low-copy-number TEs are often misannotated as genes, giving rise to as much as two-fold over estimations of gene numbers [7]. This type of overestimation in gene number can play particular havoc with assessment of genic colinearity, as evidenced by studies in rice showing hundreds of gene differences between different races of $O$. sativa that were later shown to all be explained by mis-annotated TEs [9]. Hence, many early publications showing numerous genic exceptions to microcolinearity are incorrect because of this routine annotation error.

Sequence comparisons in closely related haplotypes in Arabidopsis, in rice and in wheat have demonstrated that unequal homologous recombination and illegitimate recombination are the major forces that remove DNA from flowering plant genomes [16, 21, 60, 90, 91]. These activities can remove $>100 \mathrm{Mb}$ of DNA from a plant genome in just one million years [62], but the rate of removal appears to be much faster in some angiosperms than in others [87]. Most of the removed DNA is derived from TEs, but other intergenic DNA and extra gene copies are also removed by these processes [60].

Several recent studies have accentuated the fact that not all genomic regions evolve at the same rate. Disease resistance gene clusters are known to be unstable even in map position [55], and to also undergo high rates of unequal recombination [76], including some recombination events that are delimited to specific sites that can optimize novel pathogen recognition specificities [70]. Ribosomal RNA gene clusters also appear to vary in map position even in close relatives [25]. Perhaps most surprising, the composition and arrangement of sequences in centromeres have been found to be hyper-variable, primarily by the 
process of unequal homologous recombination [61, 63, 65]. This rapid rearrangement by recombination in a region that is deficient in crossovers suggests a very tight control over the outcomes of recombination, especially a powerful bias toward non-crossover, intrastrand and/or sister chromatid outcomes [61]. This core centromeric instability has been argued to yield centromeres that have the potential to outcompete other centromeres for choice as the germinal nucleus in egg development [66].

In summary, local sequence comparisons of closely related grass genomes and of intraspecific haplotypes have begun to reveal the major mechanisms driving genome evolution. These include gene and genome duplication, gene silencing and eventual deletion of duplicated genes subsequent to polyploidization, transposable element amplification, gene movement mediated by transposition of mobile elements, unequal homologous recombination, and illegitimate recombination. All of these processes are quite variable even when comparing closely related species, so their differences in levels of activity (and, possibly, specificity) are responsible for the very different genomes found in flowering plants.

\section{The past, present, and future of plant genome comparisons}

Perhaps the most valuable insight gained from comparative genomic analyses in rice and related grasses has been the astounding instability of genome structure against a fairly conserved set of biological functions. As mentioned above, at a local genome level, two maize plants are often more different from each other than a human is from a chimpanzee, or even from a macaque. The grasses and other angiosperms obviously insulate their gene functions from the great majority of this genome change, in manners that we do not now understand at even the most minimal level.

As shown in Drosophila, pursuit of full genome analyses in several species within a dense phylogenetic framework can be exceptionally productive $[18,79]$. In plants, the Oryza genus provides such a unique opportunity to investigate various aspects of gene and genome evolution with the availability of a robust phylogenetic framework [31,97], rich genomic resources [5, 49], and a near-perfect reference genome [41]. The ongoing sequence comparisons in the Oryza genus will provide dramatic and lineage-oriented insights into the creation of new genes, the evolution of gene structure and function, conserved non-coding sequences, the evolutionary dynamics of duplicated gene in polyploid species, centromere drive and a wealth of other issues.

As maize genome sequencing nears completion of its first draft, whole genome comparison of maize and rice will provide an unprecedented opportunity to study grass genome function and evolution. Because maize is derived from a fairly recent tetraploid [80], identifying the homoeologous segments and subsequent comparisons of these segments will illustrate how genome duplication has shaped the maize genome, and reveal the evolutionary fate of this type of duplicated gene $[47,94]$. Because all grass genomes are derived from a shared paleopolyploid [71, 83, 95], identication and comparison of two sets of homoeologous chromosomal segments in rice and four sets of homoeologous chromosomal segments in maize will reveal common and lineage-specific patterns of conservation [77], suggest mechanisms for gene movement [40, 53], and possibly identify signatures of cases where these movements led to significant biological outcomes.

The exciting next few years of grass genome comparative genomics, with great emphasis on the Oryzae and on maize and its relatives (e.g., sorghum and sugarcane), will provide a framework for the next generation of plant genome analyses. At the technical level, comparative genome analysis on a few model species like rice, maize, sorghum, and Brachypodium has opened up avenues to the highly leveraged study of any other grass. No single species is more enriched for "interesting" genes than any other species, but the traditional tractability of studying these interesting genes was centered on the model species with excellent molecular, physiological, biochemical, cell biological and genetic toolkits. Because of comparative genomics, this historical limitation no longer holds true.

With highly conserved gene content across the grasses, small-genome surrogates (or, even better, those surrogates with sequenced genomes) can be used to provide facile access to any shared grass gene. Moreover, the discovery of novel genes or modified gene functions that make each species unique can now be performed by simple EST analysis or trait mapping. Once these candidate genes for family- or genus- or species-specific gene functions are identified, they can now be easily isolated and tested for the ability to condition novel biological function by introduction into easily-transformed model species.

Despite, perhaps because of, the many important discoveries that have been made over the last 15-20 years of plant comparative genomics, we have more questions to answer now than we did at the outset. Because of the continued extraordinary increases in throughput and decreases in cost of nucleic acid sequence analyses, many more plant species will be investigated with a much broader (and better-conceived) set of phylogenetic justifications. Genetic maps, physical maps and EST analyses are all needed for hundreds or thousands of plant species to identify shared and novel traits. Every one of these genes can be tested for function in a few model species (by forward genetic, reverse genetic and transgenic technologies), so the 
orthologues, paralogues and "new" genes can also be compared and "uncovered" in a conductive genetic background or backgrounds. With such torrents of data on the horizon, better tools for sorting the gold from the grit will be needed. We have no doubt that the plant science community is up to this task, and that rice will continue its exceptional comparative genomic contributions to this ongoing golden age of plant biology.

Acknowledgements The writing of this manuscript was supported by grants from the National Science Foundation of the USA (\#0501814 to JLB) and the Chinese Academy of Sciences (\# KSCX2-YW-N-028 to MC).

\section{References}

1. Adams KL, Wendel JF. Polyploidy and genome evolution in plants. Curr Opin Plant Biol 2005;8:135-41.

2. Adams MD, Celniker SE, Holt RA, Evans CA, Gocayne JD, et al. The genome sequence of Drosophila melanogaster. Science 2000;287:2185-95

3. Ahn S, Anderson JA, Sorrells ME, Tanksley SD. Homoeologous relationships of rice, wheat and maize chromosomes. Mol Gen Genet 1993;241:483-90.

4. Ahn S, Tanksley SD. Comparative linkage maps of the rice and maize genomes. Proc Natl Acad Sci USA 1993;90:7980-4.

5. Ammiraju JS, Luo M, Goicoechea JL, Wang W, Kudrna D, et al. The Oryza bacterial artificial chromosome library resource: construction and analysis of 12 deep-coverage large-insert BAC libraries that represent the 10 genome types of the genus Oryza. Genome Res 2006;16:140-7.

6. Ammiraju JS, Zuccolo A, Yu Y, Song X, Piegu B, et al. Evolutionary dynamics of an ancient retrotransposon family provides insights into evolution of genome size in the genus Oryza. Plant J 2007;52:342-51.

7. Bennetzen JL, Coleman C, Liu R, Ma J, Ramakrishna W. Consistent over-estimation of gene number in complex plant genomes. Curr Opin Plant Biol 2004;7:732-6.

8. Bennetzen JL, Freeling M. Grasses as a single genetic system: genome composition, collinearity and compatibility. Trends Genet 1993;9:259-61.

9. Bennetzen JL, Ma J. The genetic colinearity of rice and other cereals on the basis of genomic sequence analysis. Curr Opin Plant Biol 2003;6:128-33.

10. Bennetzen JL, Ma J, Devos KM. Mechanisms of recent genome size variation in flowering plants. Ann Bot (Lond) 2005;95:127-32.

11. Bonierbale MW, Plaisted RL, Tanksley SD. RFLP maps based on a common set of clones reveal modes of chromosomal evolution in potato and tomato. Genetics 1988;120:1095-103.

12. Bowers JE, Arias MA, Asher R, Avise JA, Ball RT, et al. Comparative physical mapping links conservation of microsynteny to chromosome structure and recombination in grasses. Proc Natl Acad Sci USA 2005;102:13206-11.

13. Bremer K. Gondwanan evolution of the grass alliance of families (Poales). Evolution 2002;56:1374-87.

14. Brueggeman R, Rostoks N, Kudrna D, Kilian A, Han F, Chen J, et al. The barley stem rust-resistance gene Rpgl is a novel diseaseresistance gene with homology to receptor kinases. Proc Natl Acad Sci USA 2002;99:9328-33.

15. Chantret N, Salse J, Sabot F, Bellec A, Laubin B, et al. Contrasted microcolinearity and gene evolution within a homoeologous region of wheat and barley species. J Mol Evol 2008;66:138-50.
16. Chantret N, Salse J, Sabot F, Rahman S, Bellec A, et al. Molecular basis of evolutionary events that shaped the hardness locus in diploid and polyploid wheat species (Triticum and Aegilops). Plant Cell 2005;17:1033-45.

17. Chen M, SanMiguel P, Bennetzen JL. Sequence organization and conservation in sh2/al-homologous regions of sorghum and rice. Genetics 1998;148:435-43.

18. Clark AG, Eisen MB, Smith DR, Bergman CM, Oliver B, et al. Evolution of genes and genomes on the Drosophila phylogeny. Nature 2007;450:203-18.

19. Cunff LL, Garsmeur O, Raboin LM, Pauquet J, Telismart H, et al. Diploid/polyploid syntenic shuttle mapping and haplotype-specific chromosome walking toward a rust resistance gene (brul) in highly polyploid sugarcane $(2 n \sim 12 \times \sim 115)$. Genetics 2008;180:649-60.

20. Devos KM, Atkinson MD, Chinoy CN, Harcourt RL, Koebner RMD, Liu CJ, et al. Chromosomal rearrangements in the rye genome relative to that of wheat. Theor Appl Genet 1993;85:673-80.

21. Devos KM, Brown JK, Bennetzen JL. Genome size reduction through illegitimate recombination counteracts genome expansion in Arabidopsis. Genome Res 2002;12:1075-9.

22. Devos KM, Dubcovsky J, Dvorak J, Chinoy CN, Gale MD. Structural evolution of wheat chromosomes $4 \mathrm{~A}, 5 \mathrm{~A}$, and 7B and its impact on recombination. Theor Appl Genet 1995;91:282-8.

23. Devos KM, Pittaway TS, Reynolds A, Gale MD. Comparative mapping reveals a complex relationship between the pearl millet genome and those of foxtail millet and rice. Theor Appl Genet 2000;100:190-8.

24. Doebley JF, Gaut BS, Smith BD. The molecular genetics of crop domestication. Cell 2006;127:1309-21.

25. Dubcovsky J, Dvorak J. Ribosomal RNA multigene loci: nomads of the Triticeae genomes. Genetics 1995;140:1367-77.

26. Dubcovsky J, Ramakrishna W, SanMiguel PJ, Busso CS, Yan L, Shiloff BA, et al. Comparative sequence analysis of colinear barley and rice bacterial artificial chromosomes. Plant Physiol 2001;125:1342-53.

27. Feldman M, Liu B, Segal G, Abbo S, Levy AA, Vega JM. Rapid elimination of low-copy DNA sequences in polyploid wheat: a possible mechanism for differentiation of homoeologous chromosomes. Genetics 1997;147:1381-7.

28. Feuillet C, Keller B. High gene density is conserved at syntenic loci of small and large grass genomes. Proc Natl Acad Sci USA 1999;96:8265-70.

29. Fu H, Dooner HK. Intraspecific violation of genetic colinearity and its implications in maize. Proc Natl Acad Sci USA 2002;99:9573-8

30. Gale MD, Devos KM. Plant comparative genetics after 10 years. Science 1998;282:656-9.

31. Ge S, Sang T, Lu BR, Hong DY. Phylogeny of rice genomes with emphasis on origins of allotetraploid species. Proc Natl Acad Sci USA 1999; $96: 14400-5$.

32. Gilbert W. Why genes in pieces? Nature 1978;271:501.

33. Griffiths S, Sharp R, Foote TN, Bertin I, Wanous M, Reader S, et al. Molecular characterization of $P h 1$ as a major chromosome pairing locus in polyploid wheat. Nature 2006;439:749-52.

34. Gu YQ, Coleman-Derr D, Kong X, Anderson OD. Rapid genome evolution revealed by comparative sequence analysis of orthologous regions from four Triticeae genomes. Plant Physiol 2004;135:459-70.

35. Guimaraes CT, Sills GR, Sobral BW. Comparative mapping of Andropogoneae: Saccharum L. (sugarcane) and its relation to sorghum and maize. Proc Natl Acad Sci USA 1997;94:14261-6.

36. Guo H, Moose SP. Conserved noncoding sequences among cultivated cereal genomes identify candidate regulatory sequence elements and patterns of promoter evolution. Plant Cell 2003;15:1143-58. 
37. Han F, Kilian A, Chen JP, Kudrna D, Steffenson B, Yamamoto K, et al. Sequence analysis of a rice BAC covering the syntenous barley Rpg1 region. Genome 1999;42:1071-6.

38. Hulbert SH, Richter TE, Axtell JD, Bennetzen JL. Genetic mapping and characterization of sorghum and related crops by means of maize DNA probes. Proc Natl Acad Sci USA 1990;87:4251-5.

39. Hurles M. Gene duplication: the genomic trade in spare parts. PLoS Biol 2004;2:E206.

40. Ilic K, SanMiguel PJ, Bennetzen JL. A complex history of rearrangement in an orthologous region of the maize, sorghum, and rice genomes. Proc Natl Acad Sci USA 2003;100:12265-70.

41. IRGSP. The map-based sequence of the rice genome. Nature 2005;436:793-800

42. Jannoo N, Grivet L, Chantret N, Garsmeur O, Glaszmann JC, Arruda $\mathrm{P}$, et al. Orthologous comparison in a gene-rich region among grasses reveals stability in the sugarcane polyploid genome. Plant J 2007;50:574-85.

43. Jiang N, Bao Z, Zhang X, Eddy SR, Wessler SR. Pack-MULE transposable elements mediate gene evolution in plants. Nature 2004;431:569-73.

44. Kapitonov VV, Jurka J. Rolling-circle transposons in eukaryotes. Proc Natl Acad Sci USA 2001;98:8714-9.

45. Kaplinsky NJ, Braun DM, Penterman J, Goff SA, Freeling M. Utility and distribution of conserved noncoding sequences in the grasses. Proc Natl Acad Sci USA 2002;99:6147-51.

46. Kashkush K, Feldman M, Levy AA. Gene loss, silencing and activation in a newly synthesized wheat allotetraploid. Genetics 2002;160:1651-9.

47. Kellis M, Birren BW, Lander ES. Proof and evolutionary analysis of ancient genome duplication in the yeast Saccharomyces cerevisiae. Nature 2004;428:617-24.

48. Kellogg EA. Evolutionary history of the grasses. Plant Physiol 2001;125:1198-205.

49. Kim H, Hurwitz B, Yu Y, Collura K, Gill N, SanMiguel P, et al. Construction, alignment and analysis of 12 framework physical maps that represent the 10 genome types of the genus Oryza. Genome Biol 2008;9:R45.

50. Kim H, SanMiguel P, Nelson W, Collura K, Wissotski M, Walling JG, et al. Comparative physical mapping between Oryza sativa (AA genome type) and O. punctata (BB genome type). Genetics 2007;176:379-90.

51. Kim JS, Islam-Faridi MN, Klein PE, Stelly DM, Price HJ, Klein RR, et al. Comprehensive molecular cytogenetic analysis of sorghum genome architecture: distribution of euchromatin, heterochromatin, genes and recombination in comparison to rice. Genetics 2005; 171:1963-76.

52. Lai J, Li Y, Messing J, Dooner HK. Gene movement by Helitron transposons contributes to the haplotype variability of maize. Proc Natl Acad Sci USA 2005;102:9068-73.

53. Lai J, Ma J, Swigonova Z, Ramakrishna W, Linton E, Llaca V, et al. Gene loss and movement in the maize genome. Genome Res 2004:14:1924-31.

54. Lal SK, Giroux MJ, Brendel V, Vallejos CE, Hannah LC. The maize genome contains a helitron insertion. Plant Cell 2003;15:381-91.

55. Leister D, Kurth J, Laurie DA, Yano M, Sasaki T, Devos K, et al. Rapid reorganization of resistance gene homologues in cereal genomes. Proc Natl Acad Sci USA 1998;95:370-5.

56. Lescot M, Piffanelli P, Ciampi AY, Ruiz M, Blanc G, et al. Insights into the Musa genome: syntenic relationships to rice and between Musa species. BMC Genomics 2008;9:58.

57. Lin R, Ding L, Casola C, Ripoll DR, Feschotte C, Wang H. Transposase-derived transcription factors regulate light signaling in Arabidopsis. Science 2007;318:1302-5.
58. Liu H, Sachidanandam R, Stein L. Comparative genomics between rice and Arabidopsis shows scant collinearity in gene order. Genome Res 2001;11:2020-6.

59. Lukens LN, Pires JC, Leon E, Vogelzang R, Oslach L, Osborn T. Patterns of sequence loss and cytosine methylation within a population of newly resynthesized Brassica napus allopolyploids. Plant Physiol 2006;140:336-48.

60. Ma J, Bennetzen JL. Rapid recent growth and divergence of rice nuclear genomes. Proc Natl Acad Sci USA 2004;101:12404-10.

61. Ma J, Bennetzen JL. Recombination, rearrangement, reshuffling, and divergence in a centromeric region of rice. Proc Natl Acad Sci USA 2006;103:383-8.

62. Ma J, Devos KM, Bennetzen JL. Analyses of LTR-retrotransposon structures reveal recent and rapid genomic DNA loss in rice. Genome Res 2004;14:860-9.

63. Ma J, Jackson SA. Retrotransposon accumulation and satellite amplification mediated by segmental duplication facilitate centromere expansion in rice. Genome Res 2006;16:251-9.

64. Ma J, SanMiguel P, Lai J, Messing J, Bennetzen JL. DNA rearrangement in orthologous orp regions of the maize, rice and sorghum genomes. Genetics 2005;170:1209-20.

65. Ma J, Wing RA, Bennetzen JL, Jackson SA. Plant centromere organization: a dynamic structure with conserved functions. Trends Genet 2007;23:134-9.

66. Malik HS, Henikoff S. Conflict begets complexity: the evolution of centromeres. Curr Opin Genet Dev 2002;12:711-8.

67. Moore G, Devos KM, Wang Z, Gale MD. Cereal genome evolution, Grasses, line up and form a circle. Curr Biol 1995;5:737-9.

68. Morgante M, Brunner S, Pea G, Fengler K, Zuccolo A, Rafalski A. Gene duplication and exon shuffling by helitron-like transposons generate intraspecies diversity in maize. Nat Genet 2005;37:997-1002.

69. Nagamura Y. Conservation of duplicated segments between rice chromosome 11 and chromosome 12. Breeding Science 1995;45:373-6.

70. Nagy ED, Bennetzen JL. Pathogen corruption and site-directed recombination at a plant disease resistance gene cluster. Genome Res. 2008; doi:10.1101/gr.078766.108.

71. Paterson AH, Bowers JE, Chapman BA. Ancient polyploidization predating divergence of the cereals, and its consequences for comparative genomics. Proc Natl Acad Sci USA 2004;101:9903-8.

72. Paterson AH, Lin YR, Li Z, Schertz KF, Doebley JF, Pinson SR, et al. Convergent domestication of cereal crops by independent mutations at corresponding genetic loci. Science 1995;269:1714-8.

73. Peng J, Richards DE, Hartley NM, Murphy GP, Devos KM, et al. 'Green revolution' genes encode mutant gibberellin response modulators. Nature 1999;400:256-61.

74. Piegu B, Guyot R, Picault N, Roulin A, Saniyal A, Kim H, et al. Doubling genome size without polyploidization: dynamics of retrotransposition-driven genomic expansions in Oryza australiensis, a wild relative of rice. Genome Res 2006;16:1262-9.

75. Ramakrishna W, Dubcovsky J, Park YJ, Busso C, Emberton J, SanMiguel $\mathrm{P}$, et al. Different types and rates of genome evolution detected by comparative sequence analysis of orthologous segments from four cereal genomes. Genetics 2002;162:1389-400.

76. Richter TE, Pryor TJ, Bennetzen JL, Hulbert SH. New rust resistance specificities associated with recombination in the $R p 1$ complex in maize. Genetics 1995;141:373-81.

77. Salse J, Bolot S, Throude M, Jouffe V, Piegu B, Quraishi UM, et al. Identification and characterization of shared duplications between rice and wheat provide new insight into grass genome evolution. Plant Cell 2008;20:11-24.

78. SanMiguel P, Tikhonov A, Jin YK, Motchoulskaia N, Zakharov D, Melake-Berhan A, et al. Nested retrotransposons in the intergenic regions of the maize genome. Science 1996;274:765-8. 
79. Stark A, Lin MF, Kheradpour P, Pedersen JS, Parts L, et al. Discovery of functional elements in 12 Drosophila genomes using evolutionary signatures. Nature 2007;450:219-32.

80. Swigonova Z, Lai J, Ma J, Ramakrishna W, Llaca V, Bennetzen $\mathrm{JL}$, et al. Close split of sorghum and maize genome progenitors. Genome Res 2004;14:1916-23.

81. The Chimpanzee Sequencing and Analysis Consortium. Initial sequence of the chimpanzee genome and comparison with the human genome. Nature 2005;437:69-87.

82. Thomas CA Jr. The genetic organization of chromosomes. Annu Rev Genet 1971;5:237-56.

83. Tian C, Xiong Y, Liu T, Sun S, Chen L, Chen M. Evidence for an ancient whole-genome duplication event in rice and other cereals. Acta Genetica Sinica 2005;32:519-27.

84. Tikhonov AP, SanMiguel PJ, Nakajima Y, Gorenstein NM, Bennetzen JL, Avramova Z. Colinearity and its exceptions in orthologous adh regions of maize and sorghum. Proc Natl Acad Sci USA 1999;96:7409-14.

85. Venter JC, Adams MD, Myers EW, Li PW, Mural RJ, et al. The sequence of the human genome. Science 2001;291:1304-51.

86. Vicient CM, Suoniemi A, Anamthawat-Jonsson K, Tanskanen J, Beharav A, Nevo E, et al. Retrotransposon BARE-1 and its role in genome evolution in the genus Hordeum. Plant Cell 1999;11:1769-84.

87. Vitte C, Bennetzen JL. Analysis of retrotransposon structural diversity uncovers properties and propensities in angiosperm genome evolution. Proc Natl Acad Sci USA 2006;103:17638-43.

88. Wei F, Coe E, Nelson W, Bharti AK, Engler F, et al. Physical and genetic structure of the maize genome reflects its complex evolutionary history. PLoS Genet 2007;3:e123.
89. Wicker T, Stein N, Albar L, Feuillet C, Schlagenhauf E, Keller B. Analysis of a contiguous $211 \mathrm{~kb}$ sequence in diploid wheat (Triticum monococcum L.) reveals multiple mechanisms of genome evolution. Plant J 2001;26:307-16.

90. Wicker T, Yahiaoui N, Guyot R, Schlagenhauf E, Liu ZD, Dubcovsky J, et al. Rapid genome divergence at orthologous low molecular weight glutenin loci of the A and Am genomes of wheat. Plant Cell 2003;15:1186-97.

91. Wicker T, Yahiaoui N, Keller B. Illegitimate recombination is a major evolutionary mechanism for initiating size variation in plant resistance genes. Plant J 2007;51:631-41.

92. Wilson WA, Harrington SE, Woodman WL, Lee M, Sorrells ME, McCouch SR. Inferences on the genome structure of progenitor maize through comparative analysis of rice, maize and the domesticated panicoids. Genetics 1999;153:453-73.

93. Wing RA, Ammiraju JS, Luo M, Kim H, Yu Y, et al. The Oryza map alignment project: the golden path to unlocking the genetic potential of wild rice species. Plant Mol Biol 2005;59:53-62.

94. Wu Y, Zhu Z, Ma L, Chen M. The preferential retention of starch synthesis genes reveals the impact of whole-genome duplication on grass evolution. Mol Biol Evol 2008;25:1003-6.

95. Yu J, Wang J, Lin W, Li S, Li H, et al. The genomes of Oryza sativa: a history of duplications. PLoS Biol 2005;3:e38.

96. Zhang S, Gu YQ, Singh J, Coleman-Derr D, Brar DS, Jiang N, et al. New insights into Oryza genome evolution: high gene colinearity and differential retrotransposon amplification. Plant Mol Biol 2007;64:589-600.

97. Zou XH, Zhang FM, Zhang JG, Zang LL, Tang L, Wang J, et al. Analysis of 142 genes resolves the rapid diversification of the rice genus. Genome Biol 2008;9:R49. 